wavenumbers to rounded-up wavelengths, and also to the idiosyncracies of the compiler-curve reader. An unexpected check of consistency in this last respect was obtained from two instances in the Master Data Table where the same compound had been entered twice. The result was not altogether encouraging, and this brings me to the question of accuracy. The larger the collection of data, the less chance there is of a systematic correction being made, and each re-tabulation will retain the old errors and almost certainly introduce new ones. Both occur here, though it is difficult to say on what scale. In a familiar group of eight isomeric compounds in the Master Data Table, I found five numerical errors in the material I was able to check. Two were carried over from $H B C P$ and three were new introductions; two of these errors led to misplacements in other tables. It is surprising, too, to find the compound 'Antabue', together with several related disulphides, indexed as a distibine derivative, especially as the single genuine distibine which headed the misleading list in $H B C P$ does not appear in the Atlas.

If these criticisms seem trivial or carping, the accuracy with which the data are reproduced is of the first importance in a volume such as this. The choice of sources is another matter. In most of the large spectroscopic collections the quality is adequate to good, but the physical constants and many of the ultraviolet data are taken from miscellaneous and sometimes obsolete publications in a manner which is quite unpredictable, since it reflects the piecemeal manner in which the $H B C P$ collection has been built up over the years. As a volume it is both expensive and unwieldy $(5 \mathrm{~kg}$ !), but it is probably a worthwhile acquisition for many large research or industrial laboratories; the indexing system, its real raison d'être, has many merits and, as far as I know, no parallel. If the data could be properly checked and updated the Atlas might almost justify the comical mutual adulation of its three prefaces. $\quad$ E. A. JoHNSON

\section{Humus Chemistry}

Humic Substances in the Environment. By M. Schnitzer and S. U. Khan. Pp. vii +327. (Marcel Dekker: New York, November 1972.) \$19.50. £9.30.

THIS book would have been more accurately titled "The Chemistry of Humic Substances" and one suspects that the chosen title was designed to court the interest of some environmentalists other than those schooled in chemistry. Such will find morsels to interest them in the first and last chapters but the strong meat of the work lies in the intervening pages, which are principally devoted to chemical aspects of the extraction, composition, and properties of humic substances. Surprisingly, the first brief introductory chapter makes no mention of the vast deposits of peat that constitute the main bulk of the humus compounds of the natural environment in the northern hemisphere.

In the following six chapters, however, the authors have compiled an excellent detailed systematic account of the physico-chemical properties and composition of humic substances from a wealth of information that has hitherto been scattered through various journals, reviews and diverse publications. Chapter 2 covers the extraction, fractionation and purification of humic substances and leads on logically to one dealing with chemical and another with physical methods for their characterization, followed by a fifth chapter devoted to the chemical structure of these compounds. The researches of $\mathrm{Dr}$ Schnitzer and his co-workers over the past three decades have contributed very significantly to the story, so it is natural that these are well covered-but not to the neglect of other contributors. Chapters 6 and 7 comprise very useful reviews of the reactions of humic substances with metal ions, hydrous oxides and clay minerals, again with the emphasis on laboratory studies though implications are drawn regarding the contributions of humus substances to the overall properties of natural soils.

The last chapter deals briefly with the reactions of humic compounds with synthetic organic compounds that are increasingly being added to soils for the protection of crops from pests, diseases and weeds, and with nitrogen containing substances such as fertilizer urea. Some attention is also given to the physiological effects of humus compounds on the growth of higher plants. The main topics covered in this last chapter are dealt with in greater detail in other texts of the same series of publications on soils and the environment.

Those engaged in serious research on humic substances will be grateful to the authors for providing a valuable source book of information and making a brave attempt to present a coherent story even though it adheres rather firmly to an examination of the composition and properties of the traditional "fulvic" and "humic" acid fractions without defining the "purity" of these materials adequately in some cases.

The price is high for a work of this kind. Those enthusiasts among advanced students, lecturers and professional researchers who do invest in a copy will not be disappointed: others will undoubtedly recommend it for their libraries.

J. Tinsley

\section{Chromosomes in Cytology}

General Cytogenetics. By J. Sybenga. Pp. xiii +359. (North Holland: Amsterdam and London, 1972.) Dfl. 70; \$22.

Cytogenetics originated as a purely descriptive science and morphology, with a strongly quantitative bias, continues to be an important component. Having become allied with embryology, physiology, chemistry and physics, the subject has, however, developed into a wide-ranging experimental discipline impossible to cover at other than an introductory level in one relatively slim volume. The author's emphasis of classical topics gives a somewhat distorted picture of the current state of the field, and in view of the existence of several good texts covering basic cytogenetics the need for a book of this nature is questionable.

The book's main contribution lies in a comprehensive treatment of recombination, ranging from monofactorial segregation to gene conversion and incorporating data from prokaryotes and eukaryotes. Extensive coverage is given to structural and numerical chromosome variation and a chapter on genetic chromosome maps economically compiles information from viruses to higher organisms. These topics, occupying some two-thirds of the text, are concisely surveyed and successfully illustrate the relationship between cytological observation and genetic thought. The rest of the book is less satisfactory. Specialized chromosomes and deviant cytogenetic systems are summarily described. Least successful is the chapter, entitled "Background", which deals largely with current topics related to molecular cytology. Because of the variety and highly condensed form of the information, the reader is confronted with an apparently random catalogue of facts, more irritating and disturbing than informative. This important section would have profited greatly had one chapter been devoted specifically to methods, and the terminology appended in the form of a glossary.

The book is intended as an undergraduate text. Its specialization and variable level of information probably render it unsuitable for most courses and, more important, no attempt seems to have been made to lead the student from established fact to a consideration of questions which remain unanswered. While the author's preoccupation with terminology and classification makes the text somewhat tedious, the organization into subheadings facilitates 